\title{
Perinatal environmental factors and parental psychopathology were associated with risk of autism in Danish children
}

Larsson HJ, Eaton WW, Madsen KM, et al. Risk factors for autism: perinatal factors, parental psychiatric history, and socioeconomic status. Am J Epidemiol 2005;161:916-25.

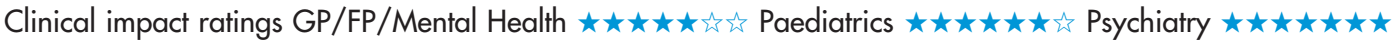

In Danish children, are perinatal factors and parental psychiatric history and social economic status associated with subsequent development of autism?

\section{METHODS}

L

Design: a case control study nested within a cohort of all children born in Denmark after 1972.

Setting: Denmark

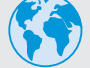

(2) Patients: 698 children (76\% boys, mean age at diagnosis 7.7 who were discharged from a Danish psychiatric hospital with a diagnosis of infantile or atypical autism before the end of December 1999 constituted the cases. For each case, 25 controls (total $n=17450$ ) were identified and individually matched to the cases by sex, birth year, and age in days.

Risk factors: perinatal risk factors including delivery and newborn characteristics (eg, fetal presentation, Apgar score at 5 $\mathrm{min}$, and gestational age at birth), pregnancy characteristics (eg, multiple gestation), and parental characteristics (eg, maternal and paternal ages; parental psychiatric history defined as presen if a psychiatric diagnosis had been recorded before the date autism was diagnosed in the child; and socioeconomic status).

$=2$ Outcome: a diagnosis of infantile or atypical autism.

\section{MAIN RESULTS}

595 cases and associated controls were included in the adjusted analyses. The risk of autism was increased for breech presentation, Apgar score $\leqslant 7$ at 5 minutes, and gestation age at birth $<35$ weeks, and for children of parents with a history of schizophrenia like psychosis or affective disorder (table).

\section{CONCLUSION}

In Danish children, perinatal environmental risk factors and parental psychiatric history were associated with subsequent development of autism. For correspondence: Dr W W Eaton, Johns Hopkins University, Baltimore, MD, USA.weaton@jhsph.edu

Sources of funding: Centers for Disease Control and Prevention; March of Dimes Birth Defects Foundation; Stanley Medical Research Institute; National Institute of Mental Health.

\section{Commentary}

The study by Larsson et al is one of several recent relatively large population-based studies investigating a possible association between obstetric complications and the risk of developing autism. ${ }^{12}$ Taken together, they present strong evidence of an association between maternal obstetric complications and an increase in risk of developing autism. The strength of the effect is modest, and none of the risk factors is specific to autism. Not surprisingly, differences exist in the individual obstetric and perinatal risk factors among studies as well as in the increase in risk conferred. A higher rate of obstetric complications is found not only in the children later diagnosed with autism, but also in their siblings without the diagnosis. Family and twin studies estimate the heritability of autism to be $>90 \%$. Autism is not necessarily caused by prenatal insults, but the observed obstetric complications may be a consequence of the same underlying genetic factors.

The study by Larsson et al is the first to include parental psychiatric history and socioeconomic status as risk factors. While socioeconomic status did not have a significant effect, a psychiatric diagnosis in the parent turned out to be the strongest risk factor. A higher rate of obstetric adversities has also been reported in pregnancies of women with a psychiatric diagnosis. ${ }^{3}$ After adjustment, parental psychiatric history remained a risk factor independent of obstetric complications. The link between a psychiatric diagnosis in the parent and autism in the child may well be due to a shared genetic susceptibility. ${ }^{4}$ The lack of diagnostic specificity across generations stresses the importance of gene-environment interactions. Large scale molecular genetic studies cutting across diagnostic boundaries and collecting information on prenatal risk factors as well as environmental factors in well defined populations may lead to a better understanding of the pathways involved.

Joachim Hallmayer, MD

Stanford University Stanford, California, USA

1 Glasson EJ, Bower C, Petterson B, et al. Perinatal factors and the development of autism: a population study. Arch Gen Psychiatry 2004;61:618-27.

2 Hultman CM, Sparén P, Cnattingius S. Perinatal risk factors for infantile autism. Epidemiology 2002;13:417-23.

3 Jablensky AV, Morgan V, Zubrick SR, et al. Pregnancy, delivery, and neonatal complications in a population cohort of women with schizophrenia and major affective disorders. Am J Psychiatry 2005;162:79-91.

4 Newschaffer CF, Cole SR. Invited commentary: Risk factors for autismperinatal factors, parental psychiatric history, and socio-economic status. Am J Epidemiol 2005;161:926-8.

Risk factors for autism in Danish children born after 1972 who were at risk until 1999*

\begin{tabular}{lll}
\hline Risk factor & Comparison & RR (95\% Cl) $\dagger$ \\
\hline Fetal presentation & Breech $v$ cephalic & $1.63(1.18$ to 2.26$)$ \\
Apgar score at 5 minutes & $1-7 v 10$ & $1.89(1.10$ to 3.27$)$ \\
Gestational age at birth (wks) & $<35 v 37-42$ & $2.45(1.55$ to 3.86$)$ \\
Parental psychiatric history & Schizophrenia-like psychosis $v$ none & $3.44(1.48$ to 7.95$)$ \\
& Affective disorder $v$ none & $2.91(1.65$ to 5.14$)$ \\
& Other $v$ none & 2.85 (2.20 to 3.69 \\
\hline
\end{tabular}

* $R R=$ relative risk. $\mathrm{Cl}$ defined in glossary.

†Adjusted model including perinatal factors, parental psychiatric history, and socioeconomic characteristics. 